\section{Crystal structure of $\left[1,1^{\prime}: 3^{\prime}, 1^{\prime \prime}\right.$-ter- phenyl]-2',3,3"'-tricarboxylic acid}

\section{Daniel A. Decato and Orion B. Berryman*}

Department of Chemistry and Biochemistry, University of Montana, 32 Campus Dr., Missoula, Montana 59812, USA. *Correspondence e-mail:

orion.berryman@umontana.edu

Received 16 May 2015; accepted 11 August 2015

Edited by S. V. Lindeman, Marquette University, USA

The asymmetric unit of the title compound, $\mathrm{C}_{21} \mathrm{H}_{14} \mathrm{O}_{6}$, comprises two symmetrically independent molecules that form a locally centrosymmetric hydrogen-bonded dimer, with the planes of the corresponding carboxylic acid groups rotated by $15.8(1)$ and $17.5(1)^{\circ}$ relative to those of the adjacent benzene rings. The crystal as a whole, however, exhibits a noncentrosymmetric packing, described by the polar space group $P c a 2_{1}$. The dimers form layers along the $a b$ plane, being interconnected by hydrogen bonds involving the remaining carboxylic acid groups. The plane of the central carboxylic acid group forms dihedral angles of 62.5 (1) and $63.0(1)^{\circ}$ with those of the adjacent benzene rings and functions as a hydrogen-bond donor and acceptor. As a donor, it interconnects adjacent layers, while as an acceptor it stabilizes the packing within the layers. The 'distal' carboxylic acid groups are nearly coplanar with the planes of the adjacent benzene rings, forming dihedral angles of $1.8(1)$ and $7.1(1)^{\circ}$. These groups also form intra- and inter-layer hydrogen bonds, but with 'reversed' functionality, as compared with the central carboxylic acid groups.

Keywords: crystal structure; hydrogen bonding; meta-terphenyl.

CCDC reference: 1418223

\section{Related literature}

For a detailed discussion on local centers of symmetry in the space group $P c a 2_{1}$, see: Marsh et al. (1998). For the synthesis

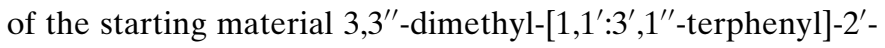
carboxylic acid, see: Du et al. (1986).

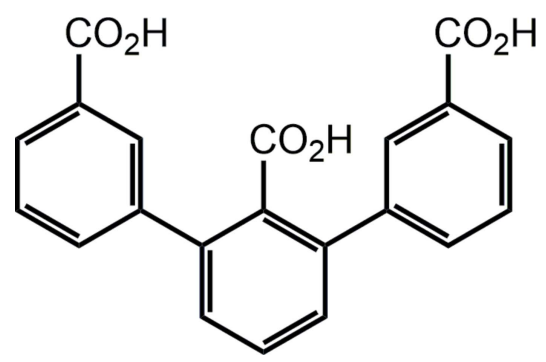

\section{Experimental}

\subsection{Crystal data}

$\mathrm{C}_{21} \mathrm{H}_{14} \mathrm{O}_{6}$

Orthorhombic, $P c a 2_{1}$

$a=23.1735$ (9) $\AA$

$b=7.2480$ (2) $\AA$

$c=20.3320(8) \AA$

$$
\begin{aligned}
& V=3415.0(2) \AA^{3} \\
& Z=8 \\
& \text { Mo } K \alpha \text { radiation } \\
& \mu=0.10 \mathrm{~mm}^{-1} \\
& T=100 \mathrm{~K} \\
& 0.3 \times 0.05 \times 0.05 \mathrm{~mm}
\end{aligned}
$$

\subsection{Data collection}

Bruker D8 VENTURE DUO diffractometer

Absorption correction: multi-scan

(SADABS; Bruker, 2012)

$T_{\text {min }}=0.695, T_{\max }=0.745$

46489 measured reflections 6467 independent reflections 5899 reflections with $I>2 \sigma(I)$ $R_{\text {int }}=0.028$

\subsection{Refinement}

$R\left[F^{2}>2 \sigma\left(F^{2}\right)\right]=0.037$

$w R\left(F^{2}\right)=0.091$

$S=1.05$

6467 reflections

511 parameters

2 restraints

$\mathrm{H}$ atoms treated by a mixture of independent and constrained refinement

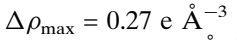

$\Delta \rho_{\min }=-0.17$ e $\AA^{-3}$

Table 1

Hydrogen-bond geometry $\left(\AA{ }^{\circ}\right)$.

\begin{tabular}{lllll}
\hline$D-\mathrm{H} \cdots A$ & $D-\mathrm{H}$ & $\mathrm{H} \cdots A$ & $D \cdots A$ & $D-\mathrm{H} \cdots A$ \\
\hline $\mathrm{O} 2-\mathrm{H} 2 \cdots \mathrm{O}^{\prime i}$ & $0.91(5)$ & $1.79(5)$ & $2.657(3)$ & $159(4)$ \\
$\mathrm{O}^{\prime}-\mathrm{H} 4 \cdots 1^{\mathrm{ii}}$ & $0.90(6)$ & $1.84(6)$ & $2.689(3)$ & $156(5)$ \\
$\mathrm{O}^{\prime}-\mathrm{H} 2^{\prime} \cdots 3^{\mathrm{iii}}$ & $0.87(6)$ & $1.76(6)$ & $2.628(3)$ & $171(5)$ \\
$\mathrm{O}^{\prime}-\mathrm{H}^{\prime} \cdots 1^{\prime i v}$ & $0.84(5)$ & $1.90(5)$ & $2.717(3)$ & $166(4)$ \\
$\mathrm{O}^{\prime}-\mathrm{H}^{\prime} \cdots \mathrm{O} 5$ & $0.92(4)$ & $1.69(4)$ & $2.593(3)$ & $168(4)$ \\
$\mathrm{O}^{\prime}-\mathrm{H} 6 \cdots 5^{\prime}$ & $1.01(3)$ & $1.58(3)$ & $2.581(3)$ & $172(6)$ \\
\hline
\end{tabular}

Symmetry codes: (i) $-x+1,-y+2, z-\frac{1}{2}$; (ii) $x, y-1, z$; (iii) $-x+\frac{3}{2}, y+1, z+\frac{1}{2}$; (iv) $x, y+1, z$.

Data collection: APEX2 (Bruker, 2012); cell refinement: SAINT (Bruker, 2012); data reduction: $S A I N T$; program(s) used to solve structure: SHELXT (Sheldrick, 2015a); program(s) used to refine structure: SHELXL2014 (Sheldrick, 2015b); molecular graphics: OLEX2 (Dolomanov et al., 2009); software used to prepare material for publication: $O L E X 2$.

\section{Acknowledgements}

This work was supported by grants from the National Science Foundation (NSF)-MRI (CHE-1337908) and the National 
Institutes of Health (CoBRE NIGMS P20GM103546). The University of Montana and the Center for Biomolecular Structure and Dynamics are aknowledged for their support of the University of Montana X-ray diffraction core facility.

Supporting information for this paper is available from the IUCr electronic archives (Reference: LD2133).

\section{References}

Bruker (2012). APEX2 and SAINT. Bruker AXS Inc., Madison, Wisconsin, USA.

Dolomanov, O. V., Bourhis, L. J., Gildea, R. J., Howard, J. A. K. \& Puschmann, H. (2009). J. Appl. Cryst. 42, 339-341.

Du, C. J. F., Hart, H. \& Ng, K. K. D. (1986). J. Org. Chem. 51, 3162-3165.

Marsh, R. E., Schomaker, V. \& Herbstein, F. H. (1998). Acta Cryst. B54, $921-$ 924.

Sheldrick, G. M. (2015a). Acta Cryst. A71, 3-8.

Sheldrick, G. M. (2015b). Acta Cryst. C71, 3-8. 


\section{supporting information}

Acta Cryst. (2015). E71, o667-o668 [https://doi.org/10.1107/S2056989015015029]

\section{Crystal structure of $\left[1,1^{\prime}: 3^{\prime}, 1^{\prime \prime}\right.$-terphenyl]-2',3,3"'-tricarboxylic acid}

\section{Daniel A. Decato and Orion B. Berryman}

\section{S1. Experimental}

\section{S1.1. Synthesis and crystallization}

3,3"-dimethyl-[1,1':3',1"-terphenyl]-2'-carboxylic acid (0.432 g, $1.4 \mathrm{mmol})$ synthesized according to Du et al. (1986). The starting material was dissolved in $15 \mathrm{ml}$ pyridine and brought to reflux. $\mathrm{KMnO}_{4}(0.50 \mathrm{~g}, 3.1 \mathrm{mmol})$ in $1 \mathrm{ml}$ of water was added, and allowed to react for 2 hours. Subsequently, 3 more additions of $\mathrm{KMnO}_{4}(0.25 \mathrm{~g}, 1.6 \mathrm{mmol})$ in $1.5 \mathrm{ml}$ of water were added every 2 hours for a total of 4 additions. After six hours, $10 \mathrm{ml}$ of water was added and the reaction was refluxed overnight. The reaction mixture was filtered while hot to remove solid $\mathrm{MnO}_{2}$. The filtrate was then concentrated under reduced pressure, and treated by $12 \mathrm{M} \mathrm{HCl}$. The resulting white precipitate was then filtered and purified via silica gel column chromatograph (50/50 hexanes/ethyl acetate with $0.5 \%$ acetic acid) to give of the title compound. $(0.366 \mathrm{~g}$ Yield 70\%) ${ }^{1} \mathrm{H}$ NMR (DMSO, $\left.400 \mathrm{MHz}\right): \delta 7.46(\mathrm{~d}, 2 \mathrm{H} J=8 \mathrm{~Hz}), 7.58(\mathrm{t}, 2 \mathrm{H}, J=8 \mathrm{~Hz}), 7.61(\mathrm{t}, 1 \mathrm{H}, J=7.2 \mathrm{~Hz}), 7.68$ (d, $2 \mathrm{H}, J=8 \mathrm{~Hz}$ ), 7.97 (d, 2H, $J=8 \mathrm{~Hz}$ ), 8.01 (s, 2H), 13.01 (s, O-H). ${ }^{13} \mathrm{C}$ NMR (DMSO, $\left.100 \mathrm{MHz}\right): \delta$ 128.4, 128.7, 129.1, $129.2,129.3,130.8,132.7,133.9,138.0,140.39,167.0,169.7$. Single crystals suitable for X-ray diffraction were obtained by vapor diffusion of hexane into an ethyl acetate solution of the title compound.

\section{S1.2. Refinement}

All $\mathrm{H}$ atoms were located in difference Fourier maps but finally their positions were determined geometrically, except for the carboxy $\mathrm{H}$ atoms that were refined with isotropic thermal parameters. The O6-H6 bond length in the carboxylic acid dimer was restrained at the distance from the corresponding residual electron density peak to the oxygen (0.996 (2) $\AA$ ) . This was done due to unreasonable lengthening $(>1.4 \AA)$ of the $\mathrm{H}-\mathrm{O}$ bond during the refinement. All other $\mathrm{H}$ atoms were refined using a riding model with fixed isotropic displacement parameters $[\mathrm{Uiso}(\mathrm{H})=1.2 \mathrm{Ueq}(\mathrm{C})$ for the $\mathrm{C}(\mathrm{H})$ groups]. Additional crystal data, data collection and structure refinement details are summarized in Table 1. 
S2. Results and discussion

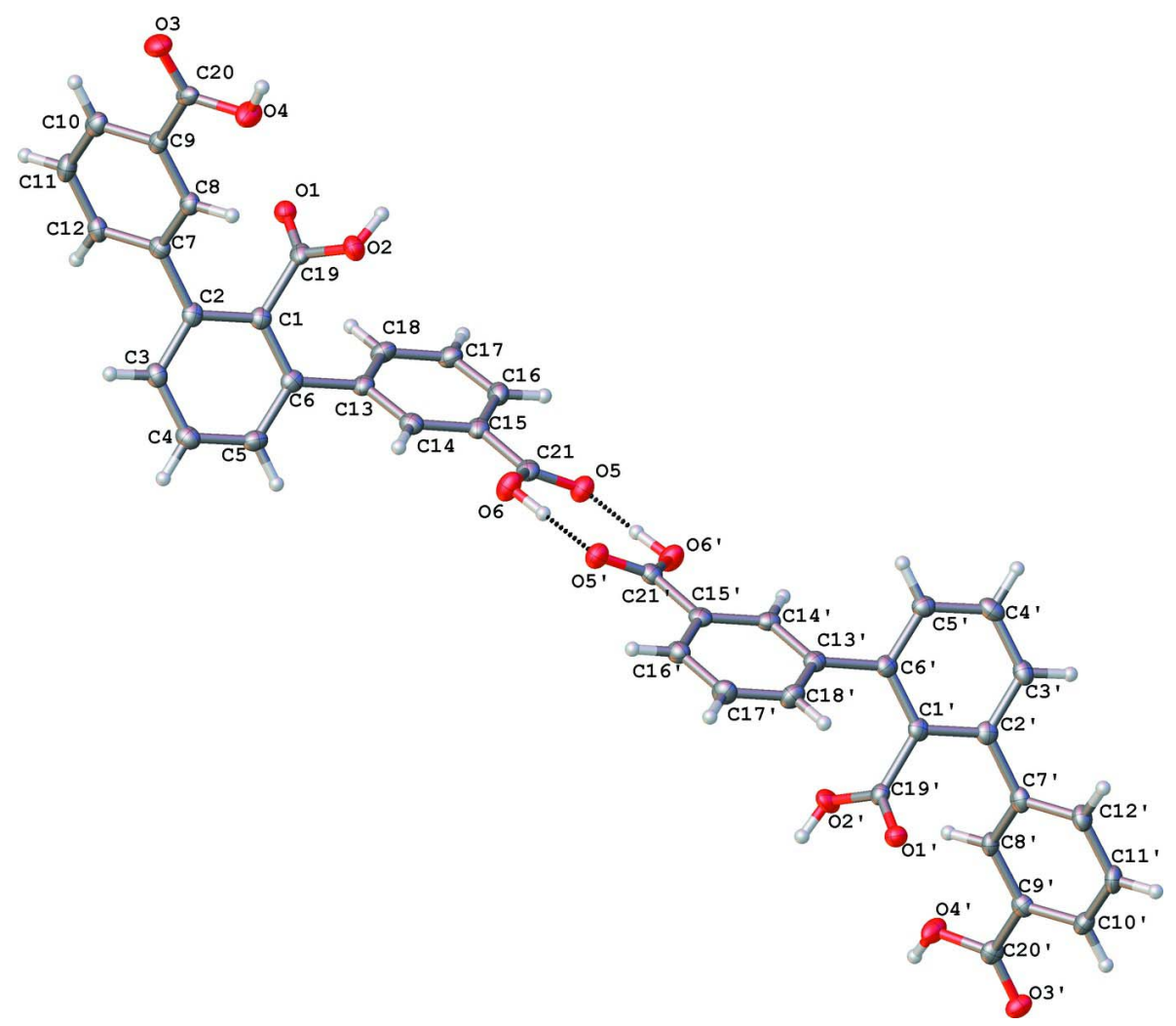

Figure 1

The asymmetric unit of the title compound, with displacement elipsoids drawn at 50\% probability level. Hydrogen atoms presented by spheres of an arbitrary radius. Intra-dimer hydrogen bonds are represented by dotted lines.

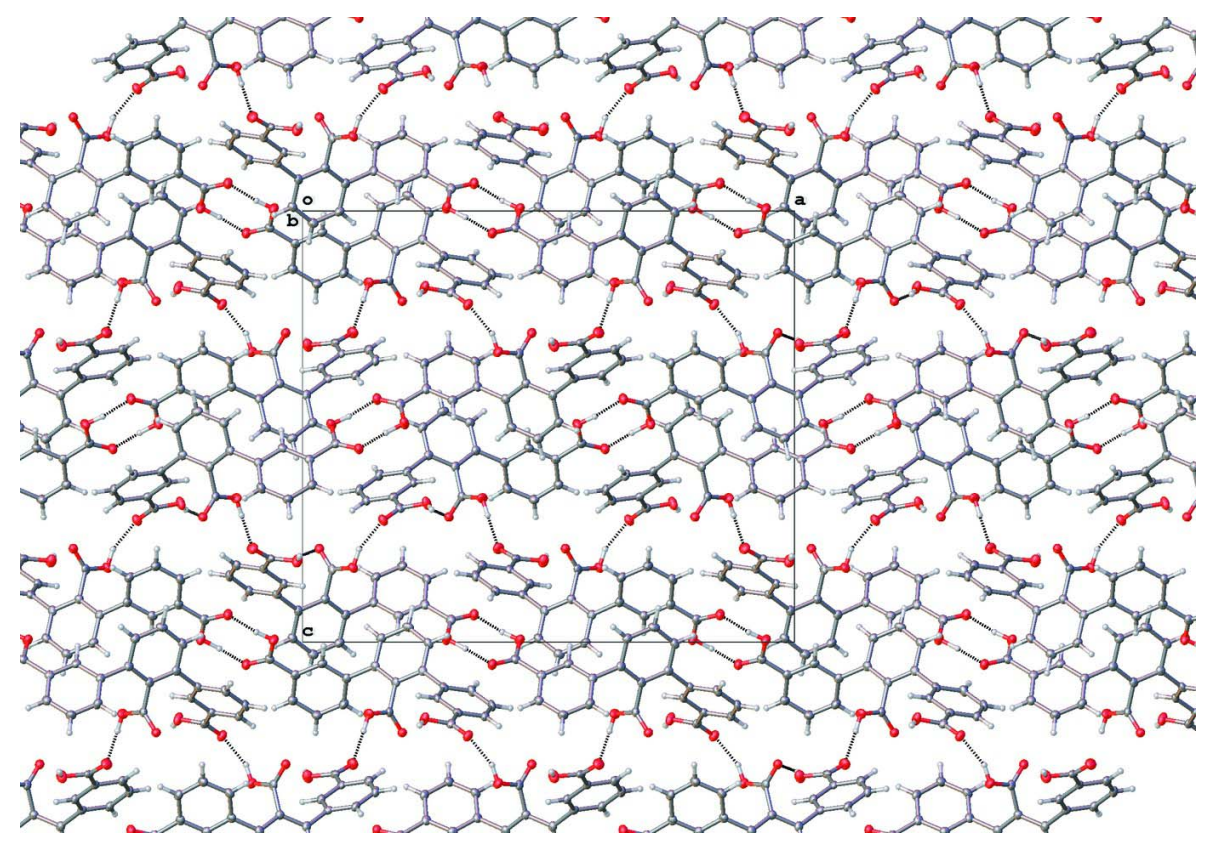


Figure 2

Packing view along $b$ axis. Hydrogen bonds are represented by dotted lines.

$\left[1,1^{\prime}: 3^{\prime}, 1^{\prime \prime}\right.$-Terphenyl]-2',3,3"'-tricarboxylic acid

Crystal data

$\mathrm{C}_{21} \mathrm{H}_{14} \mathrm{O}_{6}$

$M_{r}=362.32$

Orthorhombic, $P c a 2_{1}$

$a=23.1735(9) \AA$

$b=7.2480(2) \AA$

$c=20.3320(8) \AA$

$V=3415.0(2) \AA^{3}$

$Z=8$

$F(000)=1504$

$D_{\mathrm{x}}=1.409 \mathrm{Mg} \mathrm{m}^{-3}$

Mo $K \alpha$ radiation, $\lambda=0.71073 \AA$

Cell parameters from 9141 reflections

$\theta=3.1-25.7^{\circ}$

$\mu=0.10 \mathrm{~mm}^{-1}$

$T=100 \mathrm{~K}$

Needle, clear

$0.3 \times 0.05 \times 0.05 \mathrm{~mm}$

Data collection

Bruker D8 VENTURE DUO

diffractometer

Radiation source: sealed tube, fine-focus

TRIUMPH graphite monochromator

Detector resolution: 10.5 pixels $\mathrm{mm}^{-1}$

$\omega$ and $\varphi$ scans

Absorption correction: multi-scan

(SADABS; Bruker, 2012)

$T_{\min }=0.695, T_{\max }=0.745$

46489 measured reflections

6467 independent reflections

5899 reflections with $I>2 \sigma(I)$

$R_{\text {int }}=0.028$

$\theta_{\text {max }}=25.7^{\circ}, \theta_{\min }=3.0^{\circ}$

$h=-28 \rightarrow 28$

$k=-7 \rightarrow 8$

$l=-24 \rightarrow 24$

Refinement

Refinement on $F^{2}$

Least-squares matrix: full

$R\left[F^{2}>2 \sigma\left(F^{2}\right)\right]=0.037$

$w R\left(F^{2}\right)=0.091$

$S=1.05$

6467 reflections

511 parameters

2 restraints

Primary atom site location: structure-invariant direct methods

Hydrogen site location: mixed

$\mathrm{H}$ atoms treated by a mixture of independent and constrained refinement

$w=1 /\left[\sigma^{2}\left(F_{\mathrm{o}}^{2}\right)+(0.0518 P)^{2}+1.0855 P\right]$

where $P=\left(F_{\mathrm{o}}^{2}+2 F_{\mathrm{c}}^{2}\right) / 3$

$(\Delta / \sigma)_{\max }<0.001$

$\Delta \rho_{\max }=0.27 \mathrm{e}^{-3}$

$\Delta \rho_{\min }=-0.17 \mathrm{e} \AA^{-3}$

Special details

Experimental. SADABS-2012/1 (Bruker,2012) was used for absorption correction. wR2(int) was 0.0509 before and 0.0455 after correction. The Ratio of minimum to maximum transmission is 0.9326 . The $\lambda / 2$ correction factor is 0.0015 .

Geometry. All e.s.d.'s (except the e.s.d. in the dihedral angle between two 1.s. planes) are estimated using the full covariance matrix. The cell e.s.d.'s are taken into account individually in the estimation of e.s.d.'s in distances, angles and torsion angles; correlations between e.s.d.'s in cell parameters are only used when they are defined by crystal symmetry. An approximate (isotropic) treatment of cell e.s.d.'s is used for estimating e.s.d.'s involving 1.s. planes.

Fractional atomic coordinates and isotropic or equivalent isotropic displacement parameters $\left(\AA^{2}\right)$

\begin{tabular}{lllll}
\hline & $x$ & $y$ & $z$ & $U_{\text {iso }} * / U_{\text {eq }}$ \\
\hline O1 & $0.96578(9)$ & $0.7615(3)$ & $0.28358(11)$ & $0.0228(5)$ \\
O2 & $0.89715(9)$ & $0.5814(3)$ & $0.32756(11)$ & $0.0244(5)$ \\
H2 & $0.8852(17)$ & $0.572(5)$ & $0.285(2)$ & $0.042(11)^{*}$
\end{tabular}




\begin{tabular}{|c|c|c|c|c|}
\hline $\mathrm{O} 3$ & $1.10167(9)$ & $0.0388(3)$ & $0.28278(11)$ & $0.0303(5)$ \\
\hline $\mathrm{O} 4$ & $1.01069(10)$ & $0.0942(3)$ & $0.31188(12)$ & $0.0306(5)$ \\
\hline $\mathrm{H} 4$ & $1.006(2)$ & $-0.025(8)$ & $0.301(3)$ & $0.085(18)^{*}$ \\
\hline O5 & $0.65152(9)$ & $0.9096(3)$ & $0.43665(11)$ & $0.0258(5)$ \\
\hline O6 & $0.70682(9)$ & $0.7133(3)$ & $0.49305(12)$ & $0.0285(5)$ \\
\hline $\mathrm{C} 1$ & $0.95397(12)$ & $0.7560(4)$ & $0.40061(15)$ & $0.0188(6)$ \\
\hline $\mathrm{C} 2$ & $1.00836(13)$ & $0.7148(4)$ & $0.42736(15)$ & $0.0200(6)$ \\
\hline $\mathrm{C} 3$ & $1.02024(14)$ & $0.7721(4)$ & $0.49135(16)$ & $0.0214(7)$ \\
\hline $\mathrm{H} 3$ & 1.0572 & 0.7495 & 0.5098 & $0.026^{*}$ \\
\hline $\mathrm{C} 4$ & $0.97881(14)$ & $0.8613(4)$ & $0.52817(16)$ & $0.0235(7)$ \\
\hline $\mathrm{H} 4 \mathrm{~A}$ & 0.9872 & 0.8968 & 0.5721 & $0.028 *$ \\
\hline $\mathrm{C} 5$ & $0.92531(13)$ & $0.8995(4)$ & $0.50187(15)$ & $0.0228(7)$ \\
\hline H5 & 0.8969 & 0.9586 & 0.5282 & $0.027^{*}$ \\
\hline C6 & $0.91246(13)$ & $0.8522(4)$ & $0.43706(15)$ & $0.0197(6)$ \\
\hline $\mathrm{C} 7$ & $1.05206(13)$ & $0.6053(4)$ & $0.38977(15)$ & $0.0188(6)$ \\
\hline $\mathrm{C} 8$ & $1.03776(13)$ & $0.4318(4)$ & $0.36459(15)$ & $0.0201(7)$ \\
\hline $\mathrm{H} 8$ & 0.9999 & 0.3841 & 0.3704 & $0.024 *$ \\
\hline C9 & $1.07890(13)$ & $0.3293(4)$ & $0.33113(14)$ & $0.0178(6)$ \\
\hline $\mathrm{C} 10$ & $1.13447(14)$ & $0.3978(4)$ & $0.32208(16)$ & $0.0239(7)$ \\
\hline $\mathrm{H} 10$ & 1.1625 & 0.3272 & 0.2991 & $0.029^{*}$ \\
\hline $\mathrm{C} 11$ & $1.14841(13)$ & $0.5694(4)$ & $0.34687(16)$ & $0.0251(7)$ \\
\hline H11 & 1.1861 & 0.6178 & 0.3404 & $0.030^{*}$ \\
\hline $\mathrm{C} 12$ & $1.10790(13)$ & $0.6712(4)$ & $0.38102(15)$ & $0.0221(6)$ \\
\hline H12 & 1.1183 & 0.7878 & 0.3988 & $0.026^{*}$ \\
\hline $\mathrm{C} 13$ & $0.85570(13)$ & $0.9045(4)$ & $0.40774(15)$ & $0.0203(6)$ \\
\hline $\mathrm{C} 14$ & $0.80514(13)$ & $0.8518(4)$ & $0.43914(15)$ & $0.0212(7)$ \\
\hline H14 & 0.8072 & 0.7839 & 0.4790 & $0.025^{*}$ \\
\hline $\mathrm{C} 15$ & $0.75168(13)$ & $0.8969(4)$ & $0.41302(16)$ & $0.0205(6)$ \\
\hline $\mathrm{C} 16$ & $0.74787(13)$ & $0.9963(4)$ & $0.35433(16)$ & $0.0226(7)$ \\
\hline H16 & 0.7113 & 1.0245 & 0.3357 & $0.027^{*}$ \\
\hline C17 & $0.79797(13)$ & $1.0528(4)$ & $0.32379(16)$ & $0.0243(7)$ \\
\hline H17 & 0.7959 & 1.1237 & 0.2846 & $0.029^{*}$ \\
\hline $\mathrm{C} 18$ & $0.85140(13)$ & $1.0069(4)$ & $0.34989(16)$ & $0.0233(7)$ \\
\hline H18 & 0.8855 & 1.0459 & 0.3280 & $0.028^{*}$ \\
\hline C19 & 0.94049 (13) & $0.7010(4)$ & $0.33133(15)$ & $0.0199(6)$ \\
\hline $\mathrm{C} 20$ & $1.06555(13)$ & $0.1399(4)$ & $0.30611(14)$ & $0.0209(6)$ \\
\hline $\mathrm{C} 21$ & $0.69880(13)$ & $0.8403(4)$ & $0.44841(16)$ & $0.0220(6)$ \\
\hline $\mathrm{O} 1^{\prime}$ & $0.29732(9)$ & $0.7389(3)$ & $0.70997(10)$ & $0.0213(5)$ \\
\hline $\mathrm{O} 2^{\prime}$ & $0.36530(10)$ & $0.9209(3)$ & $0.66648(12)$ & $0.0265(5)$ \\
\hline $\mathrm{H} 2^{\prime}$ & $0.376(2)$ & $0.949(7)$ & $0.706(3)$ & $0.070(15)^{*}$ \\
\hline O3' & $0.16368(9)$ & $1.4521(3)$ & $0.71744(11)$ & $0.0247(5)$ \\
\hline O4' & $0.25240(10)$ & $1.4042(3)$ & $0.67837(12)$ & $0.0282(5)$ \\
\hline $\mathrm{H} 4^{\prime}$ & $0.2621(18)$ & $1.507(7)$ & $0.693(2)$ & $0.054(13)^{*}$ \\
\hline O5' & $0.61435(9)$ & $0.6032(3)$ & $0.55199(11)$ & $0.0247(5)$ \\
\hline O6' & $0.55852(10)$ & $0.7980(3)$ & $0.49534(11)$ & $0.0271(5)$ \\
\hline $\mathrm{C} 1^{\prime}$ & $0.31076(12)$ & $0.7424(4)$ & $0.59342(15)$ & $0.0191(6)$ \\
\hline $\mathrm{C} 2^{\prime}$ & $0.25613(13)$ & $0.7813(4)$ & $0.56681(15)$ & $0.0193(6)$ \\
\hline $\mathrm{C} 3^{\prime}$ & $0.24393(13)$ & $0.7237(4)$ & $0.50299(16)$ & $0.0216(7)$ \\
\hline
\end{tabular}




$\begin{array}{lllll}\text { H3' } & 0.2067 & 0.7449 & 0.4850 & 0.026^{*} \\ \mathrm{C}^{\prime} & 0.28570(14) & 0.6353(4) & 0.46537(16) & 0.0245(7) \\ \mathrm{H} 4^{\prime} \mathrm{A} & 0.2771 & 0.5986 & 0.4216 & 0.029^{*} \\ \mathrm{C} 5^{\prime} & 0.33996(13) & 0.6003(4) & 0.49149(16) & 0.0214(6) \\ \mathrm{H} 5^{\prime} & 0.3685 & 0.5418 & 0.4652 & 0.026^{*} \\ \mathrm{C}^{\prime} & 0.35278(13) & 0.6504(4) & 0.55605(15) & 0.0198(6) \\ \mathrm{C}^{\prime} & 0.21240(13) & 0.8890(4) & 0.60456(16) & 0.0209(7) \\ \mathrm{C} 8^{\prime} & 0.22618(12) & 1.0626(4) & 0.63010(14) & 0.0183(6) \\ \mathrm{H} 8^{\prime} & 0.2640 & 1.1105 & 0.6247 & 0.022^{*} \\ \mathrm{C} 9^{\prime} & 0.18463(13) & 1.1658(4) & 0.66341(15) & 0.0198(6) \\ \mathrm{C} 10^{\prime} & 0.12932(13) & 1.0961(4) & 0.67205(15) & 0.0212(6) \\ \mathrm{H} 10^{\prime} & 0.1011 & 1.1663 & 0.6949 & 0.025^{*} \\ \mathrm{C} 11^{\prime} & 0.11546(13) & 0.9241(4) & 0.64717(17) & 0.0242(7) \\ \mathrm{H} 11^{\prime} & 0.0777 & 0.8760 & 0.6532 & 0.029^{*} \\ \mathrm{C} 12^{\prime} & 0.15643(13) & 0.8211(4) & 0.61342(16) & 0.0228(7) \\ \mathrm{H} 12^{\prime} & 0.1464 & 0.7036 & 0.5962 & 0.027^{*} \\ \mathrm{C} 13^{\prime} & 0.41042(13) & 0.6027(4) & 0.58405(15) & 0.0198(6) \\ \mathrm{C} 14^{\prime} & 0.46007(13) & 0.6550(4) & 0.55043(15) & 0.0192(6) \\ \mathrm{H} 14^{\prime} & 0.4569 & 0.7180 & 0.5096 & 0.023^{*} \\ \mathrm{C} 15^{\prime} & 0.51443(13) & 0.6154(4) & 0.57626(15) & 0.0199(6) \\ \mathrm{C} 16^{\prime} & 0.51981(13) & 0.5223(4) & 0.63543(15) & 0.0231(7) \\ \mathrm{H} 16^{\prime} & 0.5569 & 0.4950 & 0.6528 & 0.028^{*} \\ \mathrm{C} 17^{\prime} & 0.47048(13) & 0.4694(4) & 0.66912(16) & 0.0252(7) \\ \mathrm{H} 17^{\prime} & 0.4738 & 0.4067 & 0.7100 & 0.030^{*} \\ \mathrm{C} 18^{\prime} & 0.41620(13) & 0.5075(4) & 0.64352(16) & 0.0252(7) \\ \mathrm{H} 18^{\prime} & 0.3827 & 0.4685 & 0.6666 & 0.030^{*} \\ \mathrm{C} 19^{\prime} & 0.32344(12) & 0.7990(4) & 0.66247(15) & 0.0183(6) \\ \mathrm{C} 20^{\prime} & 0.19858(13) & 1.3529(4) & 0.68902(15) & 0.0210(6) \\ \mathrm{C} 21^{\prime} & 0.56704(13) & 0.6716(4) & 0.53989(14) & 0.0197(6) \\ \mathrm{H} 6^{\prime} & 0.5939(17) & 0.824(6) & 0.477(2) & 0.045(12)^{*} \\ \mathrm{H} 6 & 0.6693(16) & 0.681(8) & 0.515(3) & 0.098(19)^{*} \\ & & & & \end{array}$

Atomic displacement parameters $\left(\AA^{2}\right)$

\begin{tabular}{lllllll}
\hline & $U^{11}$ & $U^{22}$ & $U^{33}$ & $U^{12}$ & $U^{13}$ & $U^{23}$ \\
\hline O1 & $0.0208(11)$ & $0.0242(11)$ & $0.0235(11)$ & $0.0006(8)$ & $-0.0009(9)$ & $-0.0026(9)$ \\
O2 & $0.0228(12)$ & $0.0250(11)$ & $0.0255(13)$ & $-0.0031(9)$ & $-0.0048(10)$ & $-0.0020(9)$ \\
O3 & $0.0304(12)$ & $0.0302(12)$ & $0.0304(13)$ & $0.0075(10)$ & $0.0024(10)$ & $-0.0070(10)$ \\
O4 & $0.0275(13)$ & $0.0232(12)$ & $0.0411(14)$ & $-0.0028(10)$ & $0.0041(10)$ & $-0.0084(10)$ \\
O5 & $0.0209(11)$ & $0.0256(11)$ & $0.0309(12)$ & $0.0030(9)$ & $0.0016(9)$ & $0.0029(10)$ \\
O6 & $0.0221(12)$ & $0.0292(12)$ & $0.0341(13)$ & $0.0023(10)$ & $0.0037(11)$ & $0.0100(11)$ \\
C1 & $0.0199(15)$ & $0.0150(14)$ & $0.0215(15)$ & $-0.0009(11)$ & $-0.0003(12)$ & $0.0006(11)$ \\
C2 & $0.0192(15)$ & $0.0149(13)$ & $0.0259(16)$ & $-0.0022(11)$ & $-0.0009(12)$ & $0.0019(12)$ \\
C3 & $0.0224(16)$ & $0.0195(15)$ & $0.0225(17)$ & $-0.0004(12)$ & $-0.0040(14)$ & $0.0022(13)$ \\
C4 & $0.0287(18)$ & $0.0191(15)$ & $0.0228(16)$ & $0.0006(13)$ & $-0.0033(13)$ & $-0.0010(12)$ \\
C5 & $0.0252(17)$ & $0.0213(15)$ & $0.0218(16)$ & $-0.0007(12)$ & $0.0047(13)$ & $-0.0016(13)$ \\
C6 & $0.0207(15)$ & $0.0149(14)$ & $0.0236(15)$ & $-0.0017(12)$ & $0.0019(13)$ & $0.0017(12)$ \\
C7 & $0.0217(16)$ & $0.0163(14)$ & $0.0183(15)$ & $0.0021(12)$ & $-0.0029(12)$ & $0.0022(12)$
\end{tabular}




\begin{tabular}{|c|c|c|c|c|c|c|}
\hline $\mathrm{C} 8$ & $0.0181(15)$ & $0.0199(15)$ & $0.0222(16)$ & $0.0020(12)$ & $-0.0025(12)$ & $0.0011(12)$ \\
\hline C9 & $0.0166(15)$ & $0.0189(14)$ & $0.0178(14)$ & $0.0028(11)$ & $-0.0020(12)$ & $0.0042(11)$ \\
\hline $\mathrm{C} 10$ & $0.0211(17)$ & $0.0269(16)$ & $0.0235(16)$ & $0.0045(13)$ & $-0.0009(13)$ & $0.0041(13)$ \\
\hline $\mathrm{C} 11$ & $0.0166(16)$ & $0.0287(17)$ & $0.0299(18)$ & $-0.0010(12)$ & $-0.0020(13)$ & $0.0061(14)$ \\
\hline $\mathrm{C} 12$ & $0.0203(16)$ & $0.0222(15)$ & $0.0237(16)$ & $-0.0014(12)$ & $-0.0056(13)$ & $0.0025(12)$ \\
\hline $\mathrm{C} 13$ & $0.0201(15)$ & $0.0173(14)$ & $0.0234(16)$ & $0.0029(12)$ & $0.0012(13)$ & $-0.0033(12)$ \\
\hline $\mathrm{C} 14$ & $0.0255(17)$ & $0.0171(14)$ & $0.0211(16)$ & $0.0024(12)$ & $-0.0009(13)$ & $-0.0009(12)$ \\
\hline $\mathrm{C} 15$ & $0.0206(15)$ & $0.0158(13)$ & $0.0250(16)$ & $0.0026(12)$ & $-0.0001(12)$ & $-0.0050(12)$ \\
\hline C16 & $0.0206(15)$ & $0.0211(15)$ & $0.0262(17)$ & $0.0044(13)$ & -0.0033 & $-0.0026(13)$ \\
\hline $\mathrm{C} 17$ & $0.0252(17)$ & $0.0252(15)$ & $0.0225(17)$ & $0.0048(13)$ & $0.0026(13)$ & $-0.0007(13)$ \\
\hline $\mathrm{C} 18$ & $0.0238(16)$ & $0.0227(15)$ & $0.0234(17)$ & $0.0021(14)$ & $0.0065(12)$ & $0.0007(13)$ \\
\hline C19 & $0.0173(15)$ & $0.0159(14)$ & $0.0264(16)$ & $0.0051(12)$ & $-0.0016(13)$ & $-0.0044(12)$ \\
\hline $\mathrm{C} 20$ & $0.0226(17)$ & $0.0248(15)$ & $0.0153(14)$ & $0.0081(13)$ & $-0.0009(12)$ & $0.0002(12)$ \\
\hline $\mathrm{C} 21$ & $0.0227(17)$ & $0.0170(14)$ & $0.0261(16)$ & $0.0037(12)$ & $-0.0006(13)$ & $-0.0049(12)$ \\
\hline $\mathrm{O} 1^{\prime}$ & $0.0212(11)$ & $0.0221(11)$ & $0.0207(11)$ & $-0.0001(8)$ & $0.0011(9)$ & $-0.0013(9)$ \\
\hline $\mathrm{O} 2^{\prime}$ & $0.0268(12)$ & $0.0283(11)$ & $0.0244(12)$ & $-0.0108(9)$ & $-0.0009(10)$ & $-0.0065(10)$ \\
\hline O3' & $0.0237(11)$ & $0.0205(11)$ & $0.0301(12)$ & $0.0016(9)$ & $0.0070(10)$ & $-0.0003(9)$ \\
\hline O4' & $0.0217(11)$ & $0.0201(11)$ & $0.0428(15)$ & $-0.0052(9)$ & $0.0083(10)$ & $-0.0080(11)$ \\
\hline O5' & $0.0205(12)$ & $0.0258(11)$ & $0.0277(12)$ & $0.0002(9)$ & $0.0022(9)$ & $0.0023(10)$ \\
\hline O6' & $0.0232(12)$ & $0.0300(12)$ & $0.0280(12)$ & $0.0029(9)$ & $0.0047(10)$ & $0.0076(10)$ \\
\hline $\mathrm{C} 1^{\prime}$ & $0.0191(15)$ & $0.0141(13)$ & $0.0241(16)$ & $-0.0039(11)$ & $-0.0004(13)$ & $0.0006(12)$ \\
\hline $\mathrm{C} 2^{\prime}$ & $0.0209(16)$ & $0.0136(13)$ & $0.0233(16)$ & $-0.0028(11)$ & $-0.0002(12)$ & $0.0020(12)$ \\
\hline $\mathrm{C} 3^{\prime}$ & $0.0221(16)$ & $0.0162(14)$ & $0.0264(17)$ & $-0.0011(12)$ & $-0.0064(13)$ & $0.0002(12)$ \\
\hline $\mathrm{C} 4^{\prime}$ & $0.0330(18)$ & $0.0204(16)$ & $0.0201(15)$ & $-0.0046(13)$ & $-0.0039(13)$ & $-0.0020(12)$ \\
\hline $\mathrm{C} 5^{\prime}$ & $0.0235(17)$ & $0.0177(14)$ & $0.0229(15)$ & $-0.0012(12)$ & $0.0020(13)$ & $-0.0020(12)$ \\
\hline $\mathrm{C} 6^{\prime}$ & $0.0204(16)$ & $0.0157(13)$ & $0.0234(15)$ & $-0.0037(11)$ & $0.0000(12)$ & $-0.0017(12)$ \\
\hline $\mathrm{C} 7^{\prime}$ & $0.0201(16)$ & $0.0197(14)$ & $0.0227(16)$ & $-0.0001(12)$ & $-0.0060(13)$ & $0.0049(12)$ \\
\hline $\mathrm{C} 8^{\prime}$ & $0.0140(15)$ & $0.0198(15)$ & $0.0212(16)$ & $-0.0025(11)$ & $-0.0027(12)$ & $0.0031(12)$ \\
\hline $\mathrm{C} 9^{\prime}$ & $0.0228(15)$ & $0.0182(14)$ & $0.0185(14)$ & $-0.0001(12)$ & $-0.0024(13)$ & $0.0029(12)$ \\
\hline $\mathrm{C} 10^{\prime}$ & $0.0171(15)$ & $0.0237(15)$ & $0.0227(15)$ & $0.0025(12)$ & $0.0018(13)$ & $0.0025(12)$ \\
\hline $\mathrm{C} 11^{\prime}$ & $0.0151(16)$ & $0.0265(16)$ & $0.0309(18)$ & $-0.0040(12)$ & $-0.0045(13)$ & $0.0029(14)$ \\
\hline $\mathrm{C} 12^{\prime}$ & $0.0203(16)$ & $0.0181(14)$ & $0.0301(17)$ & $-0.0029(12)$ & $-0.0057(13)$ & $-0.0005(12)$ \\
\hline C13' & $0.0217(15)$ & $0.0152(13)$ & $0.0226(16)$ & $-0.0012(12)$ & $0.0011(12)$ & $-0.0024(12)$ \\
\hline $\mathrm{C} 14^{\prime}$ & $0.0240(16)$ & $0.0155(13)$ & $0.0180(14)$ & $0.0003(11)$ & $0.0029(12)$ & $-0.0023(12)$ \\
\hline $\mathrm{C} 15^{\prime}$ & $0.0245(16)$ & $0.0146(14)$ & $0.0205(15)$ & $0.0011(12)$ & $0.0003(12)$ & $-0.0041(12)$ \\
\hline $\mathrm{C} 16^{\prime}$ & $0.0241(16)$ & $0.0230(15)$ & $0.0221(16)$ & $0.0028(13)$ & $-0.0014(12)$ & $-0.0017(12)$ \\
\hline $\mathrm{C} 17^{\prime}$ & $0.0275(17)$ & $0.0275(16)$ & $0.0207(16)$ & $0.0011(13)$ & $-0.0002(13)$ & $0.0040(14)$ \\
\hline $\mathrm{C} 18^{\prime}$ & $0.0226(16)$ & $0.0256(16)$ & $0.0272(18)$ & $0.0000(14)$ & $0.0057(13)$ & $0.0026(14)$ \\
\hline $\mathrm{C} 19^{\prime}$ & $0.0151(14)$ & $0.0174(13)$ & $0.0223(15)$ & $0.0017(11)$ & $-0.0002(13)$ & $-0.0004(12)$ \\
\hline $\mathrm{C} 20^{\prime}$ & $0.0211(16)$ & $0.0202(14)$ & $0.0217(15)$ & $-0.0021(13)$ & $0.0026(13)$ & $0.0052(12)$ \\
\hline $\mathrm{C} 21^{\prime}$ & $0.0224(16)$ & $0.0167(13)$ & $0.0198(15)$ & $-0.0017(12)$ & $-0.0019(12)$ & $-0.0023(12)$ \\
\hline
\end{tabular}

Geometric parameters $\left(\AA,{ }^{o}\right)$

\begin{tabular}{llll}
\hline $\mathrm{O} 1-\mathrm{C} 19$ & $1.216(4)$ & $\mathrm{O} 1^{\prime}-\mathrm{C} 19^{\prime}$ & $1.220(4)$ \\
$\mathrm{O} 2-\mathrm{H} 2$ & $0.91(5)$ & $\mathrm{O} 2^{\prime}-\mathrm{H} 2^{\prime}$ & $0.87(6)$ \\
$\mathrm{O} 2-\mathrm{C} 19$ & $1.329(4)$ & $\mathrm{O} 2^{\prime}-\mathrm{C} 19^{\prime}$ & $1.314(4)$ \\
$\mathrm{O} 3-\mathrm{C} 20$ & $1.209(4)$ & $\mathrm{O} 3^{\prime}-\mathrm{C} 20^{\prime}$ & $1.227(4)$
\end{tabular}




\begin{tabular}{|c|c|c|c|}
\hline $\mathrm{O} 4-\mathrm{H} 4$ & $0.90(6)$ & $\mathrm{O} 4^{\prime}-\mathrm{H} 4^{\prime}$ & $0.84(5)$ \\
\hline $\mathrm{O} 4-\mathrm{C} 20$ & $1.319(4)$ & $\mathrm{O} 4^{\prime}-\mathrm{C} 20^{\prime}$ & $1.319(4)$ \\
\hline $\mathrm{O} 5-\mathrm{C} 21$ & $1.229(4)$ & $\mathrm{O} 5^{\prime}-\mathrm{C} 21^{\prime}$ & $1.228(4)$ \\
\hline $\mathrm{O} 6-\mathrm{C} 21$ & $1.306(4)$ & $\mathrm{O} 6^{\prime}-\mathrm{C} 21^{\prime}$ & $1.303(4)$ \\
\hline O6- $-\mathrm{H} 6$ & $1.01(3)$ & $\mathrm{O} 6^{\prime}-\mathrm{H} 6^{\prime}$ & $0.92(4)$ \\
\hline $\mathrm{C} 1-\mathrm{C} 2$ & $1.405(4)$ & $\mathrm{C} 1^{\prime}-\mathrm{C} 2^{\prime}$ & $1.405(4)$ \\
\hline $\mathrm{C} 1-\mathrm{C} 6$ & $1.400(4)$ & $\mathrm{C} 1^{\prime}-\mathrm{C} 6^{\prime}$ & $1.404(4)$ \\
\hline $\mathrm{C} 1-\mathrm{C} 19$ & $1.497(4)$ & $\mathrm{C} 1^{\prime}-\mathrm{C} 19^{\prime}$ & $1.492(4)$ \\
\hline $\mathrm{C} 2-\mathrm{C} 3$ & $1.393(5)$ & $\mathrm{C} 2^{\prime}-\mathrm{C} 3^{\prime}$ & $1.392(4)$ \\
\hline $\mathrm{C} 2-\mathrm{C} 7$ & $1.496(4)$ & $\mathrm{C} 2^{\prime}-\mathrm{C} 7^{\prime}$ & $1.491(4)$ \\
\hline $\mathrm{C} 3-\mathrm{H} 3$ & 0.9500 & $\mathrm{C} 3^{\prime}-\mathrm{H} 3^{\prime}$ & 0.9500 \\
\hline $\mathrm{C} 3-\mathrm{C} 4$ & $1.378(4)$ & $\mathrm{C} 3^{\prime}-\mathrm{C} 4^{\prime}$ & $1.390(4)$ \\
\hline $\mathrm{C} 4-\mathrm{H} 4 \mathrm{~A}$ & 0.9500 & $\mathrm{C} 4^{\prime}-\mathrm{H} 4^{\prime} \mathrm{A}$ & 0.9500 \\
\hline $\mathrm{C} 4-\mathrm{C} 5$ & $1.378(4)$ & $\mathrm{C} 4^{\prime}-\mathrm{C}^{\prime}$ & $1.388(4)$ \\
\hline $\mathrm{C} 5-\mathrm{H} 5$ & 0.9500 & $\mathrm{C} 5^{\prime}-\mathrm{H} 5^{\prime}$ & 0.9500 \\
\hline $\mathrm{C} 5-\mathrm{C} 6$ & $1.394(5)$ & $\mathrm{C} 5^{\prime}-\mathrm{C} 6^{\prime}$ & $1.394(4)$ \\
\hline $\mathrm{C} 6-\mathrm{C} 13$ & $1.493(4)$ & $\mathrm{C} 6^{\prime}-\mathrm{C} 13^{\prime}$ & $1.493(4)$ \\
\hline $\mathrm{C} 7-\mathrm{C} 8$ & $1.398(4)$ & $\mathrm{C} 7^{\prime}-\mathrm{C} 8^{\prime}$ & $1.398(4)$ \\
\hline $\mathrm{C} 7-\mathrm{C} 12$ & $1.391(4)$ & $\mathrm{C} 7^{\prime}-\mathrm{C} 12^{\prime}$ & $1.399(4)$ \\
\hline $\mathrm{C} 8-\mathrm{H} 8$ & 0.9500 & $\mathrm{C} 8^{\prime}-\mathrm{H} 8^{\prime}$ & 0.9500 \\
\hline $\mathrm{C} 8-\mathrm{C} 9$ & $1.387(4)$ & $\mathrm{C} 8^{\prime}-\mathrm{C} 9^{\prime}$ & $1.395(4)$ \\
\hline $\mathrm{C} 9-\mathrm{C} 10$ & $1.393(4)$ & $\mathrm{C}^{\prime}-\mathrm{C} 10^{\prime}$ & $1.389(4)$ \\
\hline $\mathrm{C} 9-\mathrm{C} 20$ & $1.496(4)$ & $\mathrm{C} 9^{\prime}-\mathrm{C} 20^{\prime}$ & $1.488(4)$ \\
\hline $\mathrm{C} 10-\mathrm{H} 10$ & 0.9500 & $\mathrm{C} 10^{\prime}-\mathrm{H} 10^{\prime}$ & 0.9500 \\
\hline $\mathrm{C} 10-\mathrm{C} 11$ & $1.380(5)$ & $\mathrm{C} 10^{\prime}-\mathrm{C} 11^{\prime}$ & $1.384(4)$ \\
\hline C11-H11 & 0.9500 & $\mathrm{C} 11^{\prime}-\mathrm{H} 11^{\prime}$ & 0.9500 \\
\hline $\mathrm{C} 11-\mathrm{C} 12$ & $1.381(5)$ & $\mathrm{C} 11^{\prime}-\mathrm{C} 12^{\prime}$ & $1.389(5)$ \\
\hline $\mathrm{C} 12-\mathrm{H} 12$ & 0.9500 & $\mathrm{C} 12^{\prime}-\mathrm{H} 12^{\prime}$ & 0.9500 \\
\hline $\mathrm{C} 13-\mathrm{C} 14$ & $1.388(4)$ & $\mathrm{C} 13^{\prime}-\mathrm{C} 14^{\prime}$ & $1.391(4)$ \\
\hline $\mathrm{C} 13-\mathrm{C} 18$ & $1.394(5)$ & $\mathrm{C} 13^{\prime}-\mathrm{C} 18^{\prime}$ & $1.399(4)$ \\
\hline C14-H14 & 0.9500 & C14'-H14' & 0.9500 \\
\hline $\mathrm{C} 14-\mathrm{C} 15$ & $1.387(4)$ & $\mathrm{C} 14^{\prime}-\mathrm{C} 15^{\prime}$ & $1.394(4)$ \\
\hline $\mathrm{C} 15-\mathrm{C} 16$ & $1.397(5)$ & $\mathrm{C} 15^{\prime}-\mathrm{C} 16^{\prime}$ & $1.385(4)$ \\
\hline $\mathrm{C} 15-\mathrm{C} 21$ & $1.479(5)$ & $\mathrm{C} 15^{\prime}-\mathrm{C} 21^{\prime}$ & $1.483(4)$ \\
\hline $\mathrm{C} 16-\mathrm{H} 16$ & 0.9500 & $\mathrm{C} 16^{\prime}-\mathrm{H} 16^{\prime}$ & 0.9500 \\
\hline $\mathrm{C} 16-\mathrm{C} 17$ & $1.379(5)$ & $\mathrm{C} 16^{\prime}-\mathrm{C} 17^{\prime}$ & $1.387(4)$ \\
\hline $\mathrm{C} 17-\mathrm{H} 17$ & 0.9500 & $\mathrm{C} 17^{\prime}-\mathrm{H} 17^{\prime}$ & 0.9500 \\
\hline $\mathrm{C} 17-\mathrm{C} 18$ & $1.387(4)$ & $\mathrm{C} 17^{\prime}-\mathrm{C} 18^{\prime}$ & $1.389(4)$ \\
\hline $\mathrm{C} 18-\mathrm{H} 18$ & 0.9500 & $\mathrm{C} 18^{\prime}-\mathrm{H} 18^{\prime}$ & 0.9500 \\
\hline $\mathrm{C} 19-\mathrm{O} 2-\mathrm{H} 2$ & $110(3)$ & $\mathrm{C} 19^{\prime}-\mathrm{O} 2^{\prime}-\mathrm{H} 2^{\prime}$ & $115(3)$ \\
\hline $\mathrm{C} 20-\mathrm{O} 4-\mathrm{H} 4$ & $109(3)$ & $\mathrm{C} 20^{\prime}-\mathrm{O} 4^{\prime}-\mathrm{H}^{\prime}{ }^{\prime}$ & $117(3)$ \\
\hline $\mathrm{C} 21-\mathrm{O} 6-\mathrm{H} 6$ & $111(3)$ & $\mathrm{C} 21^{\prime}-\mathrm{O} 6^{\prime}-\mathrm{H} 6^{\prime}$ & $107(3)$ \\
\hline $\mathrm{C} 2-\mathrm{C} 1-\mathrm{C} 19$ & $119.7(3)$ & $\mathrm{C} 2^{\prime}-\mathrm{C} 1^{\prime}-\mathrm{C} 19^{\prime}$ & $119.0(3)$ \\
\hline $\mathrm{C} 6-\mathrm{C} 1-\mathrm{C} 2$ & $121.1(3)$ & $\mathrm{C} 6^{\prime}-\mathrm{C} 1^{\prime}-\mathrm{C} 2^{\prime}$ & $120.8(3)$ \\
\hline $\mathrm{C} 6-\mathrm{C} 1-\mathrm{C} 19$ & $119.2(3)$ & $\mathrm{C} 6^{\prime}-\mathrm{C} 1^{\prime}-\mathrm{C} 19^{\prime}$ & 120.2 \\
\hline $\mathrm{C} 1-\mathrm{C} 2-\mathrm{C} 7$ & $121.5(3)$ & $\mathrm{C} 1^{\prime}-\mathrm{C} 2^{\prime}-\mathrm{C} 7^{\prime}$ & $121.3(3)$ \\
\hline $\mathrm{C} 3-\mathrm{C} 2-\mathrm{C} 1$ & $118.4(3)$ & $\mathrm{C} 3^{\prime}-\mathrm{C} 2^{\prime}-\mathrm{C} 1^{\prime}$ & $118.8(3)$ \\
\hline
\end{tabular}




\begin{tabular}{|c|c|}
\hline $\mathrm{C} 3-\mathrm{C} 2-\mathrm{C} 7$ & $120.1(3)$ \\
\hline $\mathrm{C} 2-\mathrm{C} 3-\mathrm{H} 3$ & 119.7 \\
\hline $\mathrm{C} 4-\mathrm{C} 3-\mathrm{C} 2$ & $120.6(3)$ \\
\hline $\mathrm{C} 4-\mathrm{C} 3-\mathrm{H} 3$ & 119.7 \\
\hline $\mathrm{C} 3-\mathrm{C} 4-\mathrm{H} 4 \mathrm{~A}$ & 119.7 \\
\hline $\mathrm{C} 5-\mathrm{C} 4-\mathrm{C} 3$ & $120.7(3)$ \\
\hline $\mathrm{C} 5-\mathrm{C} 4-\mathrm{H} 4 \mathrm{~A}$ & 119.7 \\
\hline $\mathrm{C} 4-\mathrm{C} 5-\mathrm{H} 5$ & 119.7 \\
\hline $\mathrm{C} 4-\mathrm{C} 5-\mathrm{C} 6$ & $120.6(3)$ \\
\hline $\mathrm{C} 6-\mathrm{C} 5-\mathrm{H} 5$ & 119.7 \\
\hline $\mathrm{C} 1-\mathrm{C} 6-\mathrm{C} 13$ & 121.3 \\
\hline $\mathrm{C} 5-\mathrm{C} 6-\mathrm{C} 1$ & $118.4(3)$ \\
\hline $\mathrm{C} 5-\mathrm{C} 6-\mathrm{C} 13$ & 120.2 \\
\hline $\mathrm{C} 8-\mathrm{C} 7-\mathrm{C} 2$ & $120.2(3)$ \\
\hline $\mathrm{C} 12-\mathrm{C} 7-\mathrm{C} 2$ & $120.9(3)$ \\
\hline $\mathrm{C} 12-\mathrm{C} 7-\mathrm{C} 8$ & $118.9(3)$ \\
\hline $\mathrm{C} 7-\mathrm{C} 8-\mathrm{H} 8$ & 120.0 \\
\hline $\mathrm{C} 9-\mathrm{C} 8-\mathrm{C} 7$ & 119.9 \\
\hline $\mathrm{C} 9-\mathrm{C} 8-\mathrm{H} 8$ & 120.0 \\
\hline $\mathrm{C} 8-\mathrm{C} 9-\mathrm{C} 10$ & $120.6(3)$ \\
\hline $\mathrm{C} 8-\mathrm{C} 9-\mathrm{C} 20$ & $121.1(3)$ \\
\hline $\mathrm{C} 10-\mathrm{C} 9-\mathrm{C} 20$ & 118.3 \\
\hline $\mathrm{C} 9-\mathrm{C} 10-\mathrm{H} 10$ & 120.3 \\
\hline $\mathrm{C} 11-\mathrm{C} 10-\mathrm{C} 9$ & $119.3(3)$ \\
\hline $\mathrm{C} 11-\mathrm{C} 10-\mathrm{H} 10$ & 120.3 \\
\hline $\mathrm{C} 10-\mathrm{C} 11-\mathrm{H} 11$ & 119.8 \\
\hline $\mathrm{C} 10-\mathrm{C} 11-\mathrm{C} 12$ & $120.4(3)$ \\
\hline $\mathrm{C} 12-\mathrm{C} 11-\mathrm{H} 11$ & 119.8 \\
\hline $\mathrm{C} 7-\mathrm{C} 12-\mathrm{H} 12$ & 119.5 \\
\hline $\mathrm{C} 11-\mathrm{C} 12-\mathrm{C} 7$ & $120.9(3)$ \\
\hline $\mathrm{C} 11-\mathrm{C} 12-\mathrm{H} 12$ & 119.5 \\
\hline $\mathrm{C} 14-\mathrm{C} 13-\mathrm{C} 6$ & 119.3 \\
\hline $\mathrm{C} 14-\mathrm{C} 13-\mathrm{C} 18$ & $118.3(3)$ \\
\hline $\mathrm{C} 18-\mathrm{C} 13-\mathrm{C} 6$ & 122.3 \\
\hline $\mathrm{C} 13-\mathrm{C} 14-\mathrm{H} 14$ & 119.6 \\
\hline $\mathrm{C} 15-\mathrm{C} 14-\mathrm{C} 13$ & 120.9 \\
\hline $\mathrm{C} 15-\mathrm{C} 14-\mathrm{H} 14$ & 119.6 \\
\hline $\mathrm{C} 14-\mathrm{C} 15-\mathrm{C} 16$ & 120.3 \\
\hline $\mathrm{C} 14-\mathrm{C} 15-\mathrm{C} 21$ & $119.2(3)$ \\
\hline $\mathrm{C} 16-\mathrm{C} 15-\mathrm{C} 21$ & 120.4 \\
\hline $\mathrm{C} 15-\mathrm{C} 16-\mathrm{H} 16$ & 120.5 \\
\hline $\mathrm{C} 17-\mathrm{C} 16-\mathrm{C} 15$ & 119.0 \\
\hline $\mathrm{C} 17-\mathrm{C} 16-\mathrm{H} 16$ & 120.5 \\
\hline $\mathrm{C} 16-\mathrm{C} 17-\mathrm{H} 17$ & 119.7 \\
\hline $\mathrm{C} 16-\mathrm{C} 17-\mathrm{C} 18$ & 120.5 \\
\hline $\mathrm{C} 18-\mathrm{C} 17-\mathrm{H} 17$ & 119.7 \\
\hline $\mathrm{C} 13-\mathrm{C} 18-\mathrm{H} 18$ & 119.5 \\
\hline $\mathrm{C} 17-\mathrm{C} 18-\mathrm{C} 13$ & $120.9(3)$ \\
\hline
\end{tabular}

\begin{tabular}{|c|c|}
\hline $\mathrm{C} 3^{\prime}-\mathrm{C} 2^{\prime}-\mathrm{C} 7^{\prime}$ & $119.9(3)$ \\
\hline $\mathrm{C} 2^{\prime}-\mathrm{C} 3^{\prime}-\mathrm{H} 3^{\prime}$ & 119.7 \\
\hline $\mathrm{C} 4^{\prime}-\mathrm{C} 3^{\prime}-\mathrm{C} 2^{\prime}$ & $120.7(3)$ \\
\hline $\mathrm{C} 4^{\prime}-\mathrm{C} 3^{\prime}-\mathrm{H} 3^{\prime}$ & 119.7 \\
\hline $\mathrm{C} 3^{\prime}-\mathrm{C} 4^{\prime}-\mathrm{H} 4^{\prime} \mathrm{A}$ & 119.9 \\
\hline $\mathrm{C} 5^{\prime}-\mathrm{C} 4^{\prime}-\mathrm{C} 3^{\prime}$ & $120.3(3)$ \\
\hline $\mathrm{C} 5^{\prime}-\mathrm{C} 4^{\prime}-\mathrm{H} 4^{\prime} \mathrm{A}$ & 119.9 \\
\hline $\mathrm{C} 4^{\prime}-\mathrm{C} 5^{\prime}-\mathrm{H} 5^{\prime}$ & 119.8 \\
\hline $\mathrm{C} 4^{\prime}-\mathrm{C} 5^{\prime}-\mathrm{C} 6^{\prime}$ & $120.4(3)$ \\
\hline $\mathrm{C} 6^{\prime}-\mathrm{C} 5^{\prime}-\mathrm{H} 5^{\prime}$ & 119.8 \\
\hline $\mathrm{C} 1^{\prime}-\mathrm{C} 6^{\prime}-\mathrm{C} 13^{\prime}$ & $121.6(3)$ \\
\hline $\mathrm{C} 5^{\prime}-\mathrm{C} 6^{\prime}-\mathrm{C} 1^{\prime}$ & $119.1(3)$ \\
\hline $\mathrm{C} 5^{\prime}-\mathrm{C} 6^{\prime}-\mathrm{C} 13^{\prime}$ & $119.3(3)$ \\
\hline $\mathrm{C} 8^{\prime}-\mathrm{C} 7^{\prime}-\mathrm{C} 2^{\prime}$ & $120.5(3)$ \\
\hline $\mathrm{C} 8^{\prime}-\mathrm{C} 7^{\prime}-\mathrm{C} 12^{\prime}$ & $118.7(3)$ \\
\hline $\mathrm{C} 12^{\prime}-\mathrm{C} 7^{\prime}-\mathrm{C} 2^{\prime}$ & $120.8(3)$ \\
\hline $\mathrm{C} 7^{\prime}-\mathrm{C} 8^{\prime}-\mathrm{H} 8^{\prime}$ & 119.8 \\
\hline $\mathrm{C} 9^{\prime}-\mathrm{C} 8^{\prime}-\mathrm{C} 7^{\prime}$ & $120.4(3)$ \\
\hline $\mathrm{C} 9^{\prime}-\mathrm{C} 8^{\prime}-\mathrm{H} 8^{\prime}$ & 119.8 \\
\hline $\mathrm{C} 8^{\prime}-\mathrm{C} 9^{\prime}-\mathrm{C} 20^{\prime}$ & $120.6(3)$ \\
\hline $\mathrm{C} 10^{\prime}-\mathrm{C} 9^{\prime}-\mathrm{C} 8^{\prime}$ & $120.2(3)$ \\
\hline $\mathrm{C} 10^{\prime}-\mathrm{C} 9^{\prime}-\mathrm{C} 20^{\prime}$ & $119.2(3)$ \\
\hline $\mathrm{C} 9^{\prime}-\mathrm{C} 10^{\prime}-\mathrm{H} 10^{\prime}$ & 120.1 \\
\hline $\mathrm{C} 11^{\prime}-\mathrm{C} 10^{\prime}-\mathrm{C} 9^{\prime}$ & $119.7(3)$ \\
\hline $\mathrm{C} 11^{\prime}-\mathrm{C} 10^{\prime}-\mathrm{H} 10^{\prime}$ & 120.1 \\
\hline $\mathrm{C} 10^{\prime}-\mathrm{C} 11^{\prime}-\mathrm{H} 11^{\prime}$ & 119.8 \\
\hline $\mathrm{C} 10^{\prime}-\mathrm{C} 11^{\prime}-\mathrm{C} 12^{\prime}$ & $120.4(3)$ \\
\hline $\mathrm{C} 12^{\prime}-\mathrm{C} 11^{\prime}-\mathrm{H} 11^{\prime}$ & 119.8 \\
\hline $\mathrm{C} 7^{\prime}-\mathrm{C} 12^{\prime}-\mathrm{H} 12^{\prime}$ & 119.7 \\
\hline $\mathrm{C} 11^{\prime}-\mathrm{C} 12^{\prime}-\mathrm{C} 7^{\prime}$ & $120.6(3)$ \\
\hline $\mathrm{C} 11^{\prime}-\mathrm{C} 12^{\prime}-\mathrm{H} 12^{\prime}$ & 119.7 \\
\hline $\mathrm{C} 14^{\prime}-\mathrm{C} 13^{\prime}-\mathrm{C} 6^{\prime}$ & $119.3(3)$ \\
\hline $\mathrm{C} 14^{\prime}-\mathrm{C} 13^{\prime}-\mathrm{C} 18^{\prime}$ & $118.7(3)$ \\
\hline $\mathrm{C} 18^{\prime}-\mathrm{C} 13^{\prime}-\mathrm{C} 6^{\prime}$ & $122.0(3)$ \\
\hline $\mathrm{C} 13^{\prime}-\mathrm{C} 14^{\prime}-\mathrm{H} 14^{\prime}$ & 119.8 \\
\hline $\mathrm{C} 13^{\prime}-\mathrm{C} 14^{\prime}-\mathrm{C} 15^{\prime}$ & $120.4(3)$ \\
\hline $\mathrm{C} 15^{\prime}-\mathrm{C} 14^{\prime}-\mathrm{H} 14^{\prime}$ & 119.8 \\
\hline $\mathrm{C} 14^{\prime}-\mathrm{C} 15^{\prime}-\mathrm{C} 21^{\prime}$ & $119.9(3)$ \\
\hline $\mathrm{C} 16^{\prime}-\mathrm{C} 15^{\prime}-\mathrm{C} 14^{\prime}$ & $120.6(3)$ \\
\hline $\mathrm{C} 16^{\prime}-\mathrm{C} 15^{\prime}-\mathrm{C} 21^{\prime}$ & $119.5(3)$ \\
\hline $\mathrm{C} 15^{\prime}-\mathrm{C} 16^{\prime}-\mathrm{H} 16^{\prime}$ & 120.3 \\
\hline $\mathrm{C} 15^{\prime}-\mathrm{C} 16^{\prime}-\mathrm{C} 17^{\prime}$ & $119.3(3)$ \\
\hline $\mathrm{C} 17^{\prime}-\mathrm{C} 16^{\prime}-\mathrm{H} 16^{\prime}$ & 120.3 \\
\hline $\mathrm{C} 16^{\prime}-\mathrm{C} 17^{\prime}-\mathrm{H} 17^{\prime}$ & 119.8 \\
\hline $\mathrm{C} 16^{\prime}-\mathrm{C} 17^{\prime}-\mathrm{C} 18^{\prime}$ & $120.4(3)$ \\
\hline $\mathrm{C} 18^{\prime}-\mathrm{C} 17^{\prime}-\mathrm{H} 17^{\prime}$ & 119.8 \\
\hline $\mathrm{C} 13^{\prime}-\mathrm{C} 18^{\prime}-\mathrm{H} 18^{\prime}$ & 119.7 \\
\hline $\mathrm{C} 17^{\prime}-\mathrm{C} 18^{\prime}-\mathrm{C} 13^{\prime}$ & $120.6(3)$ \\
\hline
\end{tabular}




\begin{tabular}{|c|c|c|c|}
\hline $\mathrm{C} 17-\mathrm{C} 18-\mathrm{H} 18$ & 119.5 & $\mathrm{C} 17^{\prime}-\mathrm{C} 18^{\prime}-\mathrm{H} 18^{\prime}$ & 119.7 \\
\hline $\mathrm{O} 1-\mathrm{C} 19-\mathrm{O} 2$ & $123.6(3)$ & $\mathrm{O} 1^{\prime}-\mathrm{C} 19^{\prime}-\mathrm{O} 2^{\prime}$ & $123.8(3)$ \\
\hline $\mathrm{O} 1-\mathrm{C} 19-\mathrm{C} 1$ & $123.7(3)$ & $\mathrm{O} 1^{\prime}-\mathrm{C} 19^{\prime}-\mathrm{C} 1^{\prime}$ & $123.3(3)$ \\
\hline $\mathrm{O} 2-\mathrm{C} 19-\mathrm{C} 1$ & $112.7(3)$ & $\mathrm{O} 2^{\prime}-\mathrm{C} 19^{\prime}-\mathrm{C} 1^{\prime}$ & $112.9(3)$ \\
\hline $\mathrm{O} 3-\mathrm{C} 20-\mathrm{O} 4$ & $123.3(3)$ & $\mathrm{O} 3^{\prime}-\mathrm{C} 20^{\prime}-\mathrm{O} 4^{\prime}$ & $122.4(3)$ \\
\hline $\mathrm{O} 3-\mathrm{C} 20-\mathrm{C} 9$ & $123.1(3)$ & $\mathrm{O} 3^{\prime}-\mathrm{C} 20^{\prime}-\mathrm{C} 9^{\prime}$ & $123.8(3)$ \\
\hline $\mathrm{O} 4-\mathrm{C} 20-\mathrm{C} 9$ & $113.6(2)$ & $\mathrm{O} 4^{\prime}-\mathrm{C} 20^{\prime}-\mathrm{C} 9^{\prime}$ & $113.9(3)$ \\
\hline $\mathrm{O} 5-\mathrm{C} 21-\mathrm{O} 6$ & $123.4(3)$ & $\mathrm{O} 5^{\prime}-\mathrm{C} 21^{\prime}-\mathrm{O} 6^{\prime}$ & $123.9(3)$ \\
\hline $\mathrm{O} 5-\mathrm{C} 21-\mathrm{C} 15$ & $122.1(3)$ & $\mathrm{O} 5^{\prime}-\mathrm{C} 21^{\prime}-\mathrm{C} 15^{\prime}$ & $121.5(3)$ \\
\hline $\mathrm{O} 6-\mathrm{C} 21-\mathrm{C} 15$ & $114.6(3)$ & $\mathrm{O} 6^{\prime}-\mathrm{C} 21^{\prime}-\mathrm{C} 15^{\prime}$ & $114.5(3)$ \\
\hline $\mathrm{C} 1-\mathrm{C} 2-\mathrm{C} 3-\mathrm{C} 4$ & $2.2(4)$ & $\mathrm{C} 1^{\prime}-\mathrm{C} 2^{\prime}-\mathrm{C} 3^{\prime}-\mathrm{C} 4^{\prime}$ & $-2.3(4)$ \\
\hline $\mathrm{C} 1-\mathrm{C} 2-\mathrm{C} 7-\mathrm{C} 8$ & $-54.7(4)$ & $\mathrm{C} 1^{\prime}-\mathrm{C} 2^{\prime}-\mathrm{C} 7^{\prime}-\mathrm{C} 8^{\prime}$ & $54.2(4)$ \\
\hline $\mathrm{C} 1-\mathrm{C} 2-\mathrm{C} 7-\mathrm{C} 12$ & $127.4(3)$ & $\mathrm{C} 1^{\prime}-\mathrm{C} 2^{\prime}-\mathrm{C} 7^{\prime}-\mathrm{C} 12^{\prime}$ & $-127.7(3)$ \\
\hline $\mathrm{C} 1-\mathrm{C} 6-\mathrm{C} 13-\mathrm{C} 14$ & $125.4(3)$ & $\mathrm{C} 1^{\prime}-\mathrm{C} 6^{\prime}-\mathrm{C} 13^{\prime}-\mathrm{C} 14^{\prime}$ & $-127.7(3)$ \\
\hline $\mathrm{C} 1-\mathrm{C} 6-\mathrm{C} 13-\mathrm{C} 18$ & $-55.3(4)$ & $\mathrm{C} 1^{\prime}-\mathrm{C} 66^{\prime}-\mathrm{C} 13^{\prime}-\mathrm{C} 18^{\prime}$ & $52.0(4)$ \\
\hline $\mathrm{C} 2-\mathrm{C} 1-\mathrm{C} 6-\mathrm{C} 5$ & $-2.8(4)$ & $\mathrm{C} 2^{\prime}-\mathrm{C} 1^{\prime}-\mathrm{C} 6^{\prime}-\mathrm{C} 5^{\prime}$ & $1.0(4)$ \\
\hline $\mathrm{C} 2-\mathrm{C} 1-\mathrm{C} 6-\mathrm{C} 13$ & $176.9(3)$ & $\mathrm{C} 2^{\prime}-\mathrm{C} 1^{\prime}-\mathrm{C} 6^{\prime}-\mathrm{C} 13^{\prime}$ & $-178.1(3)$ \\
\hline $\mathrm{C} 2-\mathrm{C} 1-\mathrm{C} 19-\mathrm{O} 1$ & $-63.6(4)$ & $\mathrm{C} 2^{\prime}-\mathrm{C} 1^{\prime}-\mathrm{C} 19^{\prime}-\mathrm{O} 1^{\prime}$ & $62.6(4)$ \\
\hline $\mathrm{C} 2-\mathrm{C} 1-\mathrm{C} 19-\mathrm{O} 2$ & $117.5(3)$ & $\mathrm{C} 2^{\prime}-\mathrm{C} 1^{\prime}-\mathrm{C} 19^{\prime}-\mathrm{O} 2^{\prime}$ & $-117.0(3)$ \\
\hline $\mathrm{C} 2-\mathrm{C} 3-\mathrm{C} 4-\mathrm{C} 5$ & $-1.6(5)$ & $\mathrm{C} 2^{\prime}-\mathrm{C} 3^{\prime}-\mathrm{C} 4^{\prime}-\mathrm{C} 5^{\prime}$ & $1.2(4)$ \\
\hline $\mathrm{C} 2-\mathrm{C} 7-\mathrm{C} 8-\mathrm{C} 9$ & $-178.5(3)$ & $\mathrm{C} 2^{\prime}-\mathrm{C} 7^{\prime}-\mathrm{C} 8^{\prime}-\mathrm{C} 9^{\prime}$ & $177.8(3)$ \\
\hline $\mathrm{C} 2-\mathrm{C} 7-\mathrm{C} 12-\mathrm{C} 11$ & $179.4(3)$ & $\mathrm{C} 2^{\prime}-\mathrm{C} 7^{\prime}-\mathrm{C} 12^{\prime}-\mathrm{C} 11^{\prime}$ & $-178.3(3)$ \\
\hline $\mathrm{C} 3-\mathrm{C} 2-\mathrm{C} 7-\mathrm{C} 8$ & $122.8(3)$ & $\mathrm{C} 3^{\prime}-\mathrm{C} 2^{\prime}-\mathrm{C} 7^{\prime}-\mathrm{C} 8^{\prime}$ & $-123.3(3)$ \\
\hline $\mathrm{C} 3-\mathrm{C} 2-\mathrm{C} 7-\mathrm{C} 12$ & $-55.2(4)$ & $\mathrm{C} 3^{\prime}-\mathrm{C} 2^{\prime}-\mathrm{C} 7^{\prime}-\mathrm{C} 12^{\prime}$ & $54.8(4)$ \\
\hline $\mathrm{C} 3-\mathrm{C} 4-\mathrm{C} 5-\mathrm{C} 6$ & $-1.4(5)$ & $\mathrm{C} 3^{\prime}-\mathrm{C} 4^{\prime}-\mathrm{C} 5^{\prime}-\mathrm{C} 6^{\prime}$ & $1.1(4)$ \\
\hline $\mathrm{C} 4-\mathrm{C} 5-\mathrm{C} 6-\mathrm{C} 1$ & $3.5(4)$ & $\mathrm{C} 4^{\prime}-\mathrm{C} 5^{\prime}-\mathrm{C} 6^{\prime}-\mathrm{C} 1^{\prime}$ & $-2.2(4)$ \\
\hline $\mathrm{C} 4-\mathrm{C} 5-\mathrm{C} 6-\mathrm{C} 13$ & $-176.2(3)$ & $\mathrm{C} 4^{\prime}-\mathrm{C} 5^{\prime}-\mathrm{C} 6^{\prime}-\mathrm{C} 13^{\prime}$ & $176.9(3)$ \\
\hline $\mathrm{C} 5-\mathrm{C} 6-\mathrm{C} 13-\mathrm{C} 14$ & $-54.9(4)$ & $\mathrm{C} 5^{\prime}-\mathrm{C} 6^{\prime}-\mathrm{C} 13^{\prime}-\mathrm{C} 14^{\prime}$ & $53.2(4)$ \\
\hline $\mathrm{C} 5-\mathrm{C} 6-\mathrm{C} 13-\mathrm{C} 18$ & $124.4(3)$ & $\mathrm{C} 5^{\prime}-\mathrm{C} 6^{\prime}-\mathrm{C} 13^{\prime}-\mathrm{C} 18^{\prime}$ & $-127.1(3)$ \\
\hline $\mathrm{C} 6-\mathrm{C} 1-\mathrm{C} 2-\mathrm{C} 3$ & $0.0(4)$ & $\mathrm{C} 6^{\prime}-\mathrm{C} 1^{\prime}-\mathrm{C} 2^{\prime}-\mathrm{C} 3^{\prime}$ & $1.2(4)$ \\
\hline $\mathrm{C} 6-\mathrm{C} 1-\mathrm{C} 2-\mathrm{C} 7$ & $177.4(3)$ & $\mathrm{C} 6^{\prime}-\mathrm{C} 1^{\prime}-\mathrm{C} 2^{\prime}-\mathrm{C} 7^{\prime}$ & $-176.3(3)$ \\
\hline $\mathrm{C} 6-\mathrm{C} 1-\mathrm{C} 19-\mathrm{O} 1$ & $114.7(3)$ & $\mathrm{C} 6^{\prime}-\mathrm{C} 1^{\prime}-\mathrm{C} 19^{\prime}-\mathrm{O}^{\prime}$ & $-116.9(3)$ \\
\hline $\mathrm{C} 6-\mathrm{C} 1-\mathrm{C} 19-\mathrm{O} 2$ & $-64.1(3)$ & $\mathrm{C} 6^{\prime}-\mathrm{C} 1^{\prime}-\mathrm{C} 19^{\prime}-\mathrm{O} 2^{\prime}$ & $63.6(4)$ \\
\hline $\mathrm{C} 6-\mathrm{C} 13-\mathrm{C} 14-\mathrm{C} 15$ & $-179.5(3)$ & $\mathrm{C} 6^{\prime}-\mathrm{C} 13^{\prime}-\mathrm{C} 14^{\prime}-\mathrm{C} 15^{\prime}$ & $178.7(3)$ \\
\hline $\mathrm{C} 6-\mathrm{C} 13-\mathrm{C} 18-\mathrm{C} 17$ & $179.8(3)$ & $\mathrm{C} 6^{\prime}-\mathrm{C} 13^{\prime}-\mathrm{C} 18^{\prime}-\mathrm{C} 17^{\prime}$ & $-178.3(3)$ \\
\hline $\mathrm{C} 7-\mathrm{C} 2-\mathrm{C} 3-\mathrm{C} 4$ & $-175.3(3)$ & $\mathrm{C} 7^{\prime}-\mathrm{C} 2^{\prime}-\mathrm{C} 3^{\prime}-\mathrm{C} 4^{\prime}$ & $175.2(3)$ \\
\hline $\mathrm{C} 7-\mathrm{C} 8-\mathrm{C} 9-\mathrm{C} 10$ & $-0.2(4)$ & $\mathrm{C} 7^{\prime}-\mathrm{C} 8^{\prime}-\mathrm{C} 9^{\prime}-\mathrm{C} 10^{\prime}$ & $0.6(4)$ \\
\hline $\mathrm{C} 7-\mathrm{C} 8-\mathrm{C} 9-\mathrm{C} 20$ & $177.4(3)$ & $\mathrm{C} 7^{\prime}-\mathrm{C} 8^{\prime}-\mathrm{C} 9^{\prime}-\mathrm{C} 20^{\prime}$ & $-178.2(3)$ \\
\hline $\mathrm{C} 8-\mathrm{C} 7-\mathrm{C} 12-\mathrm{C} 11$ & $1.4(4)$ & $\mathrm{C} 8^{\prime}-\mathrm{C} 7^{\prime}-\mathrm{C} 12^{\prime}-\mathrm{C} 11^{\prime}$ & $-0.2(5)$ \\
\hline $\mathrm{C} 8-\mathrm{C} 9-\mathrm{C} 10-\mathrm{C} 11$ & $0.1(4)$ & $\mathrm{C} 8^{\prime}-\mathrm{C} 9^{\prime}-\mathrm{C} 10^{\prime}-\mathrm{C} 11^{\prime}$ & $-0.3(4)$ \\
\hline $\mathrm{C} 8-\mathrm{C} 9-\mathrm{C} 20-\mathrm{O} 3$ & $-172.2(3)$ & $\mathrm{C} 8^{\prime}-\mathrm{C} 9^{\prime}-\mathrm{C} 20^{\prime}-\mathrm{O}^{\prime}$ & $178.4(3)$ \\
\hline $\mathrm{C} 8-\mathrm{C} 9-\mathrm{C} 20-\mathrm{O} 4$ & $7.9(4)$ & $\mathrm{C} 8^{\prime}-\mathrm{C} 9^{\prime}-\mathrm{C} 20^{\prime}-\mathrm{O} 4^{\prime}$ & $-1.5(4)$ \\
\hline $\mathrm{C} 9-\mathrm{C} 10-\mathrm{C} 11-\mathrm{C} 12$ & $0.8(5)$ & $\mathrm{C} 9^{\prime}-\mathrm{C} 10^{\prime}-\mathrm{C} 11^{\prime}-\mathrm{C} 12^{\prime}$ & $-0.3(5)$ \\
\hline $\mathrm{C} 10-\mathrm{C} 9-\mathrm{C} 20-\mathrm{O} 3$ & $5.5(4)$ & $\mathrm{C} 10^{\prime}-\mathrm{C} 9^{\prime}-\mathrm{C} 20^{\prime}-\mathrm{O}^{\prime}$ & $-0.5(5)$ \\
\hline $\mathrm{C} 10-\mathrm{C} 9-\mathrm{C} 20-\mathrm{O} 4$ & $-174.4(3)$ & $\mathrm{C} 10^{\prime}-\mathrm{C} 9^{\prime}-\mathrm{C} 20^{\prime}-\mathrm{O}^{\prime}$ & $179.6(3)$ \\
\hline $\mathrm{C} 10-\mathrm{C} 11-\mathrm{C} 12-\mathrm{C} 7$ & $-1.5(5)$ & $\mathrm{C} 10^{\prime}-\mathrm{C} 11^{\prime}-\mathrm{C} 12^{\prime}-\mathrm{C} 7^{\prime}$ & $0.5(5)$ \\
\hline $\mathrm{C} 12-\mathrm{C} 7-\mathrm{C} 8-\mathrm{C} 9$ & $-0.5(4)$ & $\mathrm{C} 12^{\prime}-\mathrm{C} 7^{\prime}-\mathrm{C} 8^{\prime}-\mathrm{C} 9^{\prime}$ & $-0.3(4)$ \\
\hline
\end{tabular}




$\mathrm{C} 13-\mathrm{C} 14-\mathrm{C} 15-\mathrm{C} 16$
$\mathrm{C} 13-\mathrm{C} 14-\mathrm{C} 15-\mathrm{C} 21$
$\mathrm{C} 14-\mathrm{C} 13-\mathrm{C} 18-\mathrm{C} 17$
$\mathrm{C} 14-\mathrm{C} 15-\mathrm{C} 16-\mathrm{C} 17$
$\mathrm{C} 14-\mathrm{C} 15-\mathrm{C} 21-\mathrm{O} 5$
$\mathrm{C} 14-\mathrm{C} 15-\mathrm{C} 21-\mathrm{O} 6$
$\mathrm{C} 15-\mathrm{C} 16-\mathrm{C} 17-\mathrm{C} 18$
$\mathrm{C} 16-\mathrm{C} 15-\mathrm{C} 21-\mathrm{O} 5$
$\mathrm{C} 16-\mathrm{C} 15-\mathrm{C} 21-\mathrm{O} 6$
$\mathrm{C} 16-\mathrm{C} 17-\mathrm{C} 18-\mathrm{C} 13$
$\mathrm{C} 18-\mathrm{C} 13-\mathrm{C} 14-\mathrm{C} 15$
$\mathrm{C} 19-\mathrm{C} 1-\mathrm{C} 2-\mathrm{C} 3$
$\mathrm{C} 19-\mathrm{C} 1-\mathrm{C} 2-\mathrm{C} 7$
$\mathrm{C} 19-\mathrm{C} 1-\mathrm{C} 6-\mathrm{C} 5$
$\mathrm{C} 19-\mathrm{C} 1-\mathrm{C} 6-\mathrm{C} 13$
$\mathrm{C} 20-\mathrm{C} 9-\mathrm{C} 10-\mathrm{C} 11$
$\mathrm{C} 21-\mathrm{C} 15-\mathrm{C} 16-\mathrm{C} 17$

$0.1(4)$

$-179.2(3)$

$-0.9(4)$

$-1.7(4)$

$163.2(3)$

$-16.1(4)$

$1.9(5)$

$-16.1(4)$

$164.5(3)$

$-0.6(5)$

$1.1(4)$

$178.3(3)$

$-4.2(4)$

$178.9(3)$

$-1.4(4)$

$-177.6(3)$

$177.7(3)$

$\mathrm{C} 13^{\prime}-\mathrm{C} 14^{\prime}-\mathrm{C} 15^{\prime}-\mathrm{C} 16^{\prime}$
$\mathrm{C} 13^{\prime}-\mathrm{C} 14^{\prime}-\mathrm{C} 15^{\prime}-\mathrm{C} 21^{\prime}$
$\mathrm{C} 14^{\prime}-\mathrm{C} 13^{\prime}-\mathrm{C} 18^{\prime}-\mathrm{C} 17^{\prime}$
$\mathrm{C} 14^{\prime}-\mathrm{C} 15^{\prime}-\mathrm{C} 16^{\prime}-\mathrm{C} 17^{\prime}$
$\mathrm{C} 14^{\prime}-\mathrm{C} 15^{\prime}-\mathrm{C} 21^{\prime}-\mathrm{O} 5^{\prime}$
$\mathrm{C} 14^{\prime}-\mathrm{C} 15^{\prime}-\mathrm{C} 21^{\prime}-\mathrm{O} 6^{\prime}$
$\mathrm{C} 15^{\prime}-\mathrm{C} 16^{\prime}-\mathrm{C} 17^{\prime}-\mathrm{C} 18^{\prime}$
$\mathrm{C} 16^{\prime}-\mathrm{C} 15^{\prime}-\mathrm{C} 21^{\prime}-\mathrm{O} 5^{\prime}$
$\mathrm{C} 16^{\prime}-\mathrm{C} 15^{\prime}-\mathrm{C} 21^{\prime}-\mathrm{O} 6^{\prime}$
$\mathrm{C} 16^{\prime}-\mathrm{C} 17^{\prime}-\mathrm{C} 18^{\prime}-\mathrm{C} 13^{\prime}$
$\mathrm{C} 18^{\prime}-\mathrm{C} 13^{\prime}-\mathrm{C} 14^{\prime}-\mathrm{C} 15^{\prime}$
$\mathrm{C} 19^{\prime}-\mathrm{C} 1^{\prime}-\mathrm{C} 2^{\prime}-\mathrm{C} 3^{\prime}$
$\mathrm{C} 19^{\prime}-\mathrm{C} 1^{\prime}-\mathrm{C} 2^{\prime}-\mathrm{C} 7^{\prime}$
$\mathrm{C} 19^{\prime}-\mathrm{C} 1^{\prime}-\mathrm{C} 6^{\prime}-\mathrm{C} 5^{\prime}$
$\mathrm{C} 19^{\prime}-\mathrm{C} 1^{\prime}-\mathrm{C} 6^{\prime}-\mathrm{C} 13^{\prime}$
$\mathrm{C} 20^{\prime}-\mathrm{C} 9^{\prime}-\mathrm{C} 10^{\prime}-\mathrm{C} 11^{\prime}$
$\mathrm{C} 21^{\prime}-\mathrm{C} 15^{\prime}-\mathrm{C} 16^{\prime}-\mathrm{C} 17^{\prime}$

$0.5(4)$

$179.9(3)$

$1.4(4)$

$-0.4(4)$

$-162.4(3)$

$18.1(4)$

$0.7(5)$

$17.0(4)$

$-162.6(3)$

$-1.2(5)$

$-1.0(4)$

$-178.2(3)$

$4.3(4)$

$-179.5(3)$

$1.3(4)$

$178.6(3)$

$-179.7(3)$

Hydrogen-bond geometry $\left(\AA,{ }^{\circ}\right)$

\begin{tabular}{|c|c|c|c|c|}
\hline$D-\mathrm{H} \cdots A$ & $D-\mathrm{H}$ & $\mathrm{H} \cdots A$ & $D \cdots A$ & $D-\mathrm{H} \cdots A$ \\
\hline $\mathrm{O} 2-\mathrm{H} 2 \cdots \mathrm{O}^{\prime \mathrm{i}}$ & $0.91(5)$ & $1.79(5)$ & $2.657(3)$ & $159(4)$ \\
\hline $\mathrm{O} 4-\mathrm{H} 4 \cdots \mathrm{O} 1^{\mathrm{ii}}$ & $0.90(6)$ & $1.84(6)$ & $2.689(3)$ & $156(5)$ \\
\hline $\mathrm{O} 2^{\prime}-\mathrm{H} 2^{\prime} \cdots \mathrm{O} 3^{\mathrm{iii}}$ & $0.87(6)$ & $1.76(6)$ & $2.628(3)$ & $171(5)$ \\
\hline $\mathrm{O} 4^{\prime}-\mathrm{H}^{\prime} \cdots \mathrm{O}^{\prime}{ }^{\prime \mathrm{iv}}$ & $0.84(5)$ & $1.90(5)$ & $2.717(3)$ & $166(4)$ \\
\hline $\mathrm{O} 6^{\prime}-\mathrm{H} 6^{\prime} \cdots \mathrm{O} 5$ & $0.92(4)$ & $1.69(4)$ & $2.593(3)$ & $168(4)$ \\
\hline $\mathrm{O} 6-\mathrm{H} 6 \cdots \mathrm{O}^{\prime}$ & $1.01(3)$ & $1.58(3)$ & 2.581 & $172(6)$ \\
\hline
\end{tabular}

Symmetry codes: (i) $-x+1,-y+2, z-1 / 2$; (ii) $x, y-1, z$; (iii) $-x+3 / 2, y+1, z+1 / 2$; (iv) $x, y+1, z$. 